\title{
Health care professionals' perspectives on the requirements facilitating the roll-out of kangaroo mother care in South Africa
}

\author{
Wilma ten Ham $^{a, *}$, Karin C.S. Minnie ${ }^{b, 1}$, Christa S.J.C. van der Walt ${ }^{b, 2}$ \\ a School of Nursing Sciences, North-West University, Potchefstroom Campus, Private Bag X6001, Potchefstroom, \\ 2520, South Africa \\ b INSINQ Focus Area, North-West University, Potchefstroom Campus, Private Bag X6001, Potchefstroom, 2520, \\ South Africa
}

\section{A R T I C L E I N F O}

Article history:

Received 21 May 2015

Accepted 27 October 2015

Available online 11 June 2016

Keywords:

Benefit levers

Best practice

Health professionals

Kangaroo mother care

Requirements

Rolling-out process

South Africa

\begin{abstract}
A B S T R A C T
Background: Using best evidence to inform practice is the cornerstone of quality patient care, and requires spread, uptake, implementation and roll-out of best practices. Kangaroo mother care (KMC) was used as a best practice which has been partly rolled-out in South Africa. In order for successful roll-out of best practices, it is important to understand what health professionals perceive as requirements for the rolling-out process. However, no published research was found on requirements for rolling-out a best practice in the South African context.

Purpose: of the research: To explore and describe the perspectives of health professionals on the requirements for the rolling-out process of KMC as a best practice in South Africa. Methodology: Twelve semi-structured individual interviews were conducted in 2012 with health professionals from various South African healthcare levels, involved in the implementation and the rolling-out process of kangaroo mother care. Content analysis were guided in terms of the four requirements for roll-out of best practices, identified in Edwards and Grinspun's Evidence Informed Model of Care.

Results: The requirements for the successful rollout of best practices mentioned by the participants in this study concur with the requirements of Edwards and Grinspun: personal alignment and protocol/policy alignment with the best practice; a roll-out plan; leadership; and supporting and reinforcing structures such as: resources, communicating, education and development regarding the best practice, and the organisational structure. The requirements were identified at four different levels: individual level (e.g. the nurse and medical specialists), management level (of the hospital), provincial level and national level.
\end{abstract}

\footnotetext{
* Corresponding author. Present address: Nelson Mandela Metropolitan University, Department of Nursing Science, Private Bag X 77000, Port Elizabeth, 6031, South Africa. Tel.: +27 0790745905 (mobile).

E-mail addresses: wilma.tenham@nmmu.ac.za, wilmatenham@gmail.com (W. ten Ham), karin.minnie@nwu.ac.za (K.C.S. Minnie), christa.vanderwalt2@gmail.com (C.S.J.C. van der Walt).

${ }^{1}$ Tel.: +27 (0) 18299 1836, +27086 2707077 (mobile).

2 Tel.: +27082 4996185 (mobile).

Peer review under responsibility of Johannesburg University.

http://dx.doi.org/10.1016/j.hsag.2015.10.005

1025-9848/@ 2015 The Authors. Publishing services by Elsevier B.V. on behalf of Johannesburg University. This is an open access article under the CC BY-NC-ND license (http://creativecommons.org/licenses/by-nc-nd/4.0/).
} 
Conclusion: Although certain requirements, such as personal alignment and reinforcing structures can be used in the roll-out of best practices, further research is desirable to promote fuller understanding of how to devise and apply the requirements in the wider adoption of best practices in South African health care settings.

(C) 2015 The Authors. Publishing services by Elsevier B.V. on behalf of Johannesburg University. This is an open access article under the CC BY-NC-ND license (http:// creativecommons.org/licenses/by-nc-nd/4.0/).

\section{Introduction}

Globally, healthcare systems are under pressure to demonstrate high quality care that is based on the best available evidence. Evidence-based or best practices in the field of nursing and midwifery aim to improve the health and health care outcomes of patients (Grol \& Grimshaw, 2003; Porter \& Teisberg, 2007), organisational outcomes and general outcomes at a health system level (Edwards \& Grinspun, 2011).

However, evidence alone is not sufficient to ensure evidence-based decision-making and requires uptake and implementation (Grol \& Grimshaw, 2003). Although evidencebased products such as best practice guidelines and best practices are developed and made available globally, the challenge remains to implement the products and then rolledout into practice (Grol, 2001; Harrison, Legare, Graham, \& Fervers, 2010). The roll-out of evidence is crucial to provide more patients with evidence-informed care (Edwards \& Grinspun, 2011).

Rolling-out, or "spreading" involves the active disseminating and implementing of best practice and knowledge used in each intervention in every care setting (Institute for Healthcare Improvement, 2008) in an organisation to the remainder of the health care system. In fact, the roll-out of evidence can only take place after successful dissemination, diffusion, adoption and implementation (Institute for Healthcare Improvement, 2008; National Health Service Institute for Innovation and Improvement, 2010).

The process of rolling-out best practices is complex. One of the crucial moments in the process is known as the tipping point, which refers to the point when individuals accept the evidence, and therefore change is inevitable (Bodenheimer, 2007). To create this tipping point there are certain requirements to speed up the process and successfully roll-out best practices throughout the healthcare system (National Health Service Institute for Innovation and Improvement, 2010).

In developing their Evidence Informed Model of Care, Edwards and Grinspun (2011) conducted a number of studies on implementing Best Practice Guidelines (BPGs). They identified four requirements to successfully roll-out best practices, the so-called "benefit levers" - alignment, permeation plans (roll-out plans), leadership for change and reinforcing and supporting structures.

The benefit levers were used to analyse health professionals' perceptions of the requirements for rolling-out Kangaroo Mother Care (KMC) as an exemplar of best practice in South Africa.
There has been much support for KMC (Bergh, Davy, Van Rooyen, Manu, Greenfield \& Participants from Ghana Health Service, 2009; Lawn, Mwansa-Kambafwile, Horta, Barros, \& Cousens, 2010; Nyqvist et al., 2010). KMC involves positioning a newborn baby vertically between the mother's breasts, promoting skin-to-skin contact, and exclusive breastfeeding (Nyqvist et al., 2010). KMC was first introduced in 1979 in San Juan de Dios hospital in Bogota, Colombia by doctors Héctor Martínez Gómez and Edgar Rey Sanabria. The practice was originally intended to modify the care of low-birth-weight infants ( $<2.5 \mathrm{~kg}$ ) (Bergh \& Pattinson, 2003; Nyqvist et al., 2010) since it improved infant development significantly, particularly that of premature infants (Nyqvist et al., 2010). KMC proved the following: it improves and extends mother-infant bonding; it reduces maternal post-partum depression; it improves parental response to infant signals; it enhances infant physiological stability and ameliorates pain in the infant; it prolongs breastfeeding (Nyqvist et al., 2010); it significantly decreases the neonatal mortality of pre-term babies (birth weight $<2 \mathrm{~kg}$ ); and it effectively reduces severe infant morbidity, predominantly from infection (Bergh et al., 2009; Lawn et al., 2010).

KMC has been widely implemented in countries such as Nigeria, Madagascar, Malawi, Ghana, Indonesia (Bergh et al., 2005, 2009) and South Africa. South Africa is taking a leading role in implementing KMC country-wide (Bergh, Van Rooyen, \& Pattinson, 2008), initially in hospitals in KwaZulu-Natal (Bergh et al., 2009; Pattinson et al., 2005), Gauteng and Mpumalanga. Major success has been achieved in implementing and rolling-out KMC in South Africa: the most recent Strategic National Plan to strengthen delivery of maternal, new-born baby, child and women's health and nutrition services (2012-2016) recommended rolling-out KMC to all public hospitals and health facilities by 2015 (National Department of Health, 2012). Much can be learnt from this exercise of rolling-out evidence-based health care practices in South Africa, which was the focus of the current study.

\subsection{Problem statement}

KMC, an example of best practice, has been implemented and rolled-out in South Africa since 1999/2000 (Bergh et al., 2005), but is still not implemented in all health settings. Based on a number of studies (Bergh et al., 2005, 2007, 2012), it can be concluded that implementing and rolling-out KMC on a large scale is complex, time-consuming and requires a variety of resources, physical space, skilled personnel and supervision, networking with healthcare authorities (local and national), 
and management support at different levels. However, no studies could be found that describe the requirements for rolling-out best practice, particularly in the South African healthcare context.

The study reported on here is believed to be the first to explore and describe the perceptions of health practitioners on the requirements for rolling-out best practices in South Africa. The benefit levers identified by Edwards and Grinspun (2011) were used as a framework to analyse perceptions of the requirements to roll-out of $\mathrm{KMC}$ as an exemplar of best practice in South Africa.

\subsection{Aims and objectives}

The aim of the study was to facilitate effective roll-out of best practices. The objective was to explore and describe the perspectives of health professionals, who were involved in the roll-out, of the requirements for rolling-out $\mathrm{KMC}$ as a best practice in South Africa.

\subsection{Definitions of key concepts}

\subsubsection{Benefit levers}

Requirements are the factors that enhance the benefits of innovations, or make them quicker or less costly to achieve (Edwards \& Grinspun, 2011).

\subsubsection{Best practice}

Best practices are related to nursing practice, methods, procedures and techniques based on high-quality evidence (products) used to obtain improved patient/health outcomes (adapted from Grol \& Grimshaw, 2003).

\subsubsection{Health professionals}

Health professionals relate or belong to a profession (Oxford English Dictionary, 2015).

\subsubsection{Rolling-out}

Rolling-out is the active dissemination of best practices and knowledge about evidence-informed interventions, and implementation of these interventions in all care settings (adapted from Institute of Healthcare Improvement, 2008).

\subsubsection{Kangaroo mother care}

Kangaroo mother care involves placing the new-born baby vertically between the mother's breasts to promote skin-toskin contact, and exclusive breastfeeding (Nyqvist et al., 2010).

\section{Material and methods}

A total of 12 semi-structured interviews were conducted in 2012 with 13 health professionals involved in implementing and rolling-out KMC at hospital/ward and provincial level in the South African healthcare system $(\mathrm{n}=12)$, and at an academic level $(n=1)$. Participants were from different provinces throughout South Africa; therefore the interviews were conducted telephonically except for one face-to-face interview. Most of the interviews were individual interviews, but one was conducted with two participants, since these participants were in the same KMC ward. The same interview schedule was used for all the interviews.

\subsection{Setting}

One interview with two participants was conducted face-toface and 11 were conducted telephonically from the NorthWest University, Potchefstroom Campus. Participants were from seven different provinces throughout South Africa.

\subsection{Procedures}

Procedures refers to recruitment, sampling and data collection.

\subsubsection{Recruitment}

Specific health professionals, who were regarded as information-rich because they were directly involved in rolling-out KMC, were contacted telephonically, or by e-mail to determine whether they were interested and willing to participate in the study. In total 13 health professionals were recruited. The researcher (XXX) asked the ones known to us to recommend other persons who could be helpful in reaching the aims of the study and then approached these individuals.

\subsubsection{Sampling}

A combination of purposive and snowball sampling was used to identify information-rich participants. Using this approach towards sampling ensured that the requirements to successfully roll-out KMC were grounded in first-hand experience.

An attempt was made to include a variety of participants from as many levels and provinces as possible, but no participants from the Eastern Cape or the North-West agreed to participate in the study. The researcher also failed to recruit anybody at hospital management level nor from the National Department of Health due to the commitments people had at those levels. However, data saturation was reached after $(n=12)$ interviews: the participants provided rich data; similar themes were repeatedly mentioned.

Table 1 outlines the sampling framework for this study.

The participants included a researcher with a healthcare background, who conducts research on implementing KMC and has published extensively, four front line nurses and five paediatricians. The participants were all working at hospital, provincial and national health system levels, and were involved in implementing and rolling-out $\mathrm{KMC}$ in South Africa.

\subsubsection{Data-collection}

XXX conducted the interviews from June until August 2012. The interview schedule involved the following questions:

- the health practitioner's involvement or role in the roll-out of KMC in South Africa,

- what was required to roll-out KMC,

- what had proved effective,

- what could have been done differently, and

- recommendations for rolling-out of best practices in the future. 
Table 1 - Sampling framework for the study.

\begin{tabular}{lllll} 
Participant number & Gender & \multicolumn{1}{c}{ Occupation } & \multicolumn{1}{c}{ Level of the health system } & \multicolumn{1}{c}{ Province } \\
\hline Participant 1 & Female & Medical doctor & Hospital kangaroo mother care ward/unit level Gauteng \\
Participant 2 & Female & Researcher with psychology background & Researcher & Gauteng \\
Participant 3 & Female & Professional nurse & Hospital kangaroo mother care ward/unit level Kwa-Zulu Natal \\
Participant 4 & Female & Professional nurse & Hospital kangaroo mother care ward/unit level Western Cape \\
Participant 5 & Female & Paediatrician & Hospital kangaroo mother care ward/unit level Western Cape \\
Participant 6 & Male & Paediatrician & Hospital kangaroo mother care ward/unit level Limpopo \\
Participant 7 & Female & Professional nurse & Hospital kangaroo mother care ward/unit level Gauteng \\
Participant 8 & Female & Paediatrician & Hospital kangaroo mother care ward/unit level Mpumalanga \\
Participant 9 & Male & Medical doctor & Hospital kangaroo mother care ward/unit level Western Cape \\
Participant 10 & Male & Paediatrician & Hospital kangaroo mother care ward/unit level Northern Cape \\
Participant 11 & Male & Paediatrician & Hospital kangaroo mother care ward/unit level Western Cape & West \\
Participant 12 & Female & Neonatologist & Provincial manager & Western Cape \\
Participant 13 & Female & Professional nurse & Provincial manager & Free State \\
\hline
\end{tabular}

Probing questions were used to obtain more information. If one interview was not sufficient to obtain the data requested, a follow-up was conducted by telephone or e-mail by mutual agreement. The telephonic and face-to-face interviews were tape recorded and field notes were kept of each interview.

\subsection{Data analysis}

XXX prepared transcriptions of the interviews and e-mailed them to the respective participants to check for accuracy. It was necessary to know the participants' identities until confirmation of the content of the trascriptions was received. Thereafter the participants' identities were deleted from the transcripts. The content of the transcriptions required minimal changes.

Data were analysed using qualitative content analysis. Content analysis was guided by the four requirements for rolling-out best practices identified in Edwards and Grinspun's (2011) Evidence Informed Model of Care.

\subsection{Ethical considerations}

The study adhered to the latest version of the Helsinki Declaration. Ethical consent was granted by the Ethics Committee of xxx University (Ethics number XXX-00005-12-A1). Although participants did not directly benefit from participating, their opinions will assist in understanding what is required to roll-out of best practices, which may improve the quality of practice.

Participation in the study was voluntary. Consent forms were sent electronically after the participants agreed to participate. The consent form explained the aims of the study and the participants were invited to ask questions. They were also informed about their right to withdraw at any time. They were informed that the transcript of their interview would be sent to each of them to ensure that the data was correctly captured, and that for this reason their identities would remain on the interview transcript until each participant had agreed that the relevant transcript was a correct version of what she/he had said. After this confirmation, the identity of the person interviewed would be removed from the transcript. Furthermore, the participants' identities would not be included in the scientific publication, or in any other form of dissemination of the findings of the study.

\subsection{Trustworthiness}

Credibility was increased in the following ways: two content specialists reviewed the interview schedule to ensure it generated data needed to answer the research question (King \& Horrocks, 2010). Furthermore, participants were provided with evidence-informed information allowing them to review the draft transcript for feedback - the so-called 'respondent validation' or 'respondent feedback' (King \& Horrocks, 2010; Simons, 2009). The interview findings were compared with concepts in the literature (Botma, Greeff, Mulaudzi \& Wright, 2010). To avoid low-quality recording and ensure a good quality transcript, the quality of the recording instrument was checked beforehand (King \& Horrocks, 2010). Objectivity was ensured as follows: when presenting the data, the researcher stayed as close to the evidence as possible and demonstrated the way in which interpretations and findings were developed (Simons, 2009).

\section{Results and findings}

The analysis identified the requirements (themes) and the nature of each requirement (sub-themes). Edwards and Grinspun's (2011) four benefit levers were used as a framework and the requirements analysed accordingly.

- Alignment

- Roll-out plan

- Leadership for change

- Supporting and reinforcing structures.

There were four different levels of requirements - the individual level (e.g. the nurse and medical specialists working at the hospitals and kangaroo mother wards), the management level (of the hospital), the provincial level (i.e. the provincial Department of Health) and the national level (i.e. the National Department of Health).

The main- and sub-requirements at the different levels are discussed in the following section. 


\subsection{Requirement 1: alignment}

It is crucial that buy-in (agreement) by all the stakeholders and alignment of structures and activities supporting the best practice should take place at all levels of the healthcare system. Table 2 outlines the sub-themes of the "Requirements for alignment" per level of the healthcare system.

Sub-themes relating to the alignment requirement are explained in the following sections.

\subsubsection{Sub-theme 1.1: level of alignment}

Personal alignment refers to agreement between persons, such as buy-in by individual stakeholders. Personal alignment was found at individual level, management level, provincial and national level.

At an individual level, the buy-in and support of the following stakeholders was crucial to successfully roll-out best practices.

Nursing staff

'The challenge was getting all the nursing staff on board. Also the consultant in charge originally.' (Participant 5)

\section{Patient and family}

'If the grandmother does not agree with you, kangaroo mother care is not going to happen.' (Participant 10)

At management level personal alignment involved the buyin of (senior) management:

'Staff was there; from the senior management down they said: 'Yes, we are going to do it.' (Participant 6)

At a provincial level, the buy-in of the provincial head was necessary to facilitate rolling-out best practices:

'And provincial head, every time we spoke he said: "Yes, kangaroo mother care must be done.' (Participant 6)

At a national level, the buy-in of the National Department of Health was crucial for rolling-out best practices:

'For provincial level we have to buy-in if it comes from national level, so the ones who drive it must be the National Department of Health.' (Participant 13).

\subsubsection{Sub-theme 1.2: protocol/policy alignment}

Policy alignment refers to the alignment of the supporting documents, for example, protocols or policies which should be aligned throughout the different levels of the health system to support the roll-out of best practices. Protocol/ policy alignment was found at management and provincial level.

At management level, the protocol of the hospital - aligned with protocols at provincial level - was seen as a facilitating factor:

'It's not a separate [provincial] protocol. It's just part of the neonatal management protocol.' (Participant 10)

At provincial level a perceived facilitator was the alignment of the provincial policy with the national policy:

'They [provincial Department of Health] will use the national policy as a basis, the national policy that are all in place. I hope by next year it will come out.' (Participant 4)

\subsection{Requirement 2: roll-out plan as a requirement for successful roll-out}

To ensure the successful and widespread adoption of best practices, a well-structured plan is required. Some institutions were planning implementation and thus followed a project's timeline.' (Bergh et al., 2005). However, in other hospitals not included in the project, no plan existed, and common sense was used:

'No, no, I think logical things and common sense, as I said, one has to explain to the management, to the nurse and the doctors, everybody, and then just gradually start and build on.' (Participant 11)

The researcher could find no national plan for rolling-out $\mathrm{KMC}$, which could explain why KMC was implemented differently at individual hospitals in different provinces. As one participant stated:

'In South Africa at the moment kangaroo mother care is practiced widely but not universally at all. There is very patchy implementation of kangaroo mother care across the country.' (Participant 9)

\subsection{Requirement 3: leadership for change}

Leadership is an essential requirement for the successful rollout of best practices. Perceived lack of leadership on several levels (individual, management and provincial) was mentioned. One participant noted:

Table 2 - Requirements of alignment per level.

\begin{tabular}{lllll} 
Sub-themes & Individual level & Management level & Provincial level & National level \\
\hline 1.1: Personal alignment & $\begin{array}{l}\text { Buy-in of the nurse, } \\
\text { patient, family }\end{array}$ & Buy-in (senior) management & Buy-in provincial head & Buy-in National \\
1.2: Protocol/policy alignment & - & $\begin{array}{l}\text { Alignment hospital protocol } \\
\text { with provincial protocol }\end{array}$ & $\begin{array}{l}\text { Alignment provincial } \\
\text { with national policy }\end{array}$ & - \\
& & & & \\
\hline
\end{tabular}


Table 3 - Requirements of leadership for change per level.

\begin{tabular}{|c|c|c|c|c|}
\hline Sub-themes & Individual level & $\begin{array}{l}\text { Management } \\
\text { level }\end{array}$ & $\begin{array}{l}\text { Provincial } \\
\text { level }\end{array}$ & $\begin{array}{l}\text { National } \\
\text { level }\end{array}$ \\
\hline 3.1: Leadership characteristic & $\begin{array}{l}\text { Suitable (stable, accepted by peers, } \\
\text { and be a role model) }\end{array}$ & - & Experienced & - \\
\hline 3.2: Tasks and roles of the leader & $\begin{array}{l}\text { Providing technical and emotional } \\
\text { support; ensuring the continuity of } \\
\text { the best practice; a convincing role, } \\
\text { an interactive role; an encouraging } \\
\text { role; an advisory role; and } \\
\text { involvement in the community }\end{array}$ & $\begin{array}{l}\text { Management to } \\
\text { understand } \\
\text { the leader's role }\end{array}$ & $\begin{array}{l}\text { A motivating/convincing } \\
\text { role; ensuring the continuity } \\
\text { of the best practice, and; } \\
\text { target the right people }\end{array}$ & - \\
\hline
\end{tabular}

'It was happening where there was a leader.' (Participant 6)

Table 3 outlines the sub-requirements for Leadership per level of 'service delivery'.

The identified sub-themes are mentioned as follows.

\subsubsection{Sub-theme 3.1: leadership characteristics}

At an individual level, participants mentioned the importance of selecting a suitable person as leader:

'They don't always think the thing through to as which is the best candidate.' (Participant 1)

A suitable leader must be stable (participants 2 and 10), accepted by peers (participant 10), and a role model (participant 10).

At a provincial level, the leader should be experienced (Participant 3).

\subsubsection{Sub-theme 3.2: tasks and roles of a leader}

At an individual level, the following tasks and roles were identified to facilitate roll-out of best practices - providing technical and emotional support, ensuring the continuity of the best practice, a convincing role, an interactive role and an advisory role, and involvement in the community. The encouraging role was emphasised:

'There's a positive side if you encourage people when they've done the right.' (Participant 6)
'Encouragement and energy is so important. And that's what the activist helps, because they got energy.' (Participant 5)

At a management level it was important that management understood the leader's role in facilitating the roll-out of best practices:

'There needs to be a designated understanding by management of that person's role.' (Participant 8)

Other tasks a leader has to fulfil at provincial level were assuming a motivating/convincing role (Participant 8), ensuring the continuity of the best practice (Participant 2), and target the right people for implementing and rolling-out the best practice in an organisation (Participant 12).

\subsection{Requirement 4: supporting and reinforcing structures}

Four sub-requirements, one per level, were identified under supporting and reinforcing structures (outlined in Table 4 below).

The sub-themes are explained below.

\subsubsection{Sub-theme 4.1 resources}

Certain resources were identified at each level. At an individual level, human resources were identified as crucial for rolling-out best practices. Experienced/senior clinicians as part of human resources were viewed as KMC facilitators:

Table 4 - Requirements of supporting and reinforcing structures per level.

\begin{tabular}{|c|c|c|c|c|}
\hline Sub-themes & Individual level & Management level & Provincial level & National level \\
\hline 4.1: Resources & $\begin{array}{l}\text { Human resources } \\
\text { (experienced/ } \\
\text { senior clinicians) }\end{array}$ & $\begin{array}{l}\text { Human resources } \\
\text { Infrastructure } \\
\text { Budget and funding } \\
\text { Equipment } \\
\text { Policies and guidelines }\end{array}$ & $\begin{array}{l}\text { Human resources } \\
\text { (district specialist team and } \\
\text { primary health care team) } \\
\text { Infrastructure } \\
\text { (existing departments) } \\
\text { Existing documents, forms, } \\
\text { information package hospital, } \\
\text { implementation programs }\end{array}$ & $\begin{array}{l}\text { Human resources } \\
\text { (national implementation } \\
\text { task team) } \\
\text { Infrastructure } \\
\text { National policy and } \\
\text { supportive strategy }\end{array}$ \\
\hline $\begin{array}{l}\text { 4.2: Education and } \\
\text { development }\end{array}$ & $\begin{array}{l}\text { Sharing knowledge } \\
\text { and training }\end{array}$ & Benchmarking visits & $\begin{array}{l}\text { Coordination and providing } \\
\text { training }\end{array}$ & Future training of staff \\
\hline 4.3: Communication & Meetings & - & 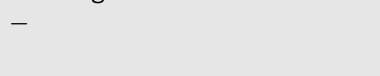 & $\begin{array}{l}\text { National awareness } \\
\text { Annual congresses }\end{array}$ \\
\hline $\begin{array}{l}\text { 4.4: Organisational } \\
\text { structure }\end{array}$ & $\begin{array}{l}\text { Multi-disciplinary } \\
\text { teamwork }\end{array}$ & $\begin{array}{l}\text { Organisational cooperation } \\
\text { and dynamics }\end{array}$ & - & - \\
\hline
\end{tabular}


'Junior people working - you need structure. If it's a senior person then you know you got the knowledge and the experience.' (Participant 1)

At management level, for successful roll-out of best practices it was important that the management provides the following support in terms of resources:

Human resources

'Because staffing norms or staffing issues were done by the management.' (Participant 7)

\section{Infrastructure}

'This would not have happened as we were not given the ward.' (Participant 1)

Budget and funding

'XXX Foundation funded a whole lot of initiatives.' (Participant 8)

Equipment

'Every year when it comes to equipment, we don't buy the sophisticated incubators.' (Participant 7)

Policies and guidelines

'There should be a protocol on this [KMC]. It has been shown to be the best way.' (Participant 5)

At provincial level, the following resources facilitated the roll-out of best practices.

\section{Human resources}

'The provincial government has now put a budget for a district specialist team, with a paediatrician in the specialist team who needs to ensure that kangaroo mother care is implemented (...) which includes community health workers, the primary health care nurse and a school nurse.' (Participant 12)

\section{Infrastructure.}

Participant 4 identified existing departments (such as Maternal Child Health (MHC) Department), existing documents/forms, existing implementation programmes, as well as existing information packages for hospitals (Participants 11 and 12) to facilitate the roll-out of kangaroo mother care.

At national level, the following resources were identified to facilitate the roll-out of best practices.

Human resources.

A national implementation task team was mentioned:

'One of the people that was on the task team here Dr. XXX, she is sitting on the national task team also.' (Participant 4)

\section{Infrastructure}

'New hospitals must be designed to care for low birth weight babies in kangaroo mother care (...) and I think that should be driven from a national level.' (Participant 10)

National policy and supportive strategies

'Develop a clear policy, with basic equipment and staffing norms for the institutions' (Participant 1)

'Continuous supporting, it's actually in the Tshwane Declaration [on breastfeeding].' (Participant 4)

3.4.2. Sub-theme 4.2: education and development

Education and development were identified at each level.

At an individual level, sharing knowledge and training was identified, participants identified certain factors that facilitated roll-out in terms of sharing knowledge and showing evidence to staff, and patients: these were training, conferences, networking, posters, surveys, and workshops (Participants 4, 7 and 11).

At management level, benchmarking visits was found a facilitator:

'One thing which helps (...) was benchmarking. Actually going to a place where it [KMC] was working.' (Participant 6).

At provincial level, providing training and coordinating training was viewed as a crucial provincial supportive task:

'To train people on kangaroo mother care. Ja, that's probably the most important.' (Participant 10)

At national level, training staff (at an individual level) in future and including best practices in the curriculum were important:

'Kangaroo mother care (...) should be part of all the learning curriculums, of all health providers, nurses and doctors.' (Participant 7)

\subsubsection{Sub-theme 4.3: communication}

Communication was identified as crucial at individual and national level.

At an individual level, meetings were mentioned as an important facilitator to evaluate the roll-out of the best practice:

'Quality circle meetings (...), that people can ask the questions and share their fears and the hiccups that you get, that you share that with each other.' (Participant 4)

At national level, national awareness campaigns and annual congresses were mentioned to communicate the best practice: 
'We have to start with mass mobilisation, media awareness, radio, adverts, newspaper articles or schools have to be taught the importance of kangaroo mother care.' (Participant 13)

'And every year, (...) we do our presentations, our research, our surveys. (...) Just to make knowledge about kangaroo mother care. Any changes, any improvement.' (Participant 7)

\subsubsection{Sub-theme 4.4: organisational structure}

Organisational structure as a requirement was identified at individual- and management level.

At an individual level, participants identified multidisciplinary team work as a facilitating factor:

'What was very good is that we had a very good team (...) the camaraderie and the sort of very close working relationship between myself, my colleagues as well as the medical side and the nurses in maternity.' (Participant 8)

'Definitely I think good relationship with your doctors, your multidisciplinary team. You must work as a team to get this well-implemented.' (Participant 4)

At management level, organisational cooperation and dynamics were pointed out as requirements for roll-out:

'The dynamics in terms of the people working together between the leaders and so on, some managed to get something with nothing.' (Participant 2)

'We had a seniority in the organisation that we could push for change.' (Participant 8)

\section{Discussion}

A summary of the results and practical implications appears below.

\subsection{Outline of the discussion}

The participants in this study identified requirements to successfully roll-out KMC that were congruent with the four main requirements facilitating roll-out of best practices identified by Edwards and Grinspun (2011). Requirements for roll-out should operate at all levels of the health care system, but in this study they were not always identified at each level. In general, the findings related to the requirements were as follows:

- It is important for the roll-out of best practices to obtain buy-in from all stakeholders at all levels (personal alignment), which is confirmed by Chin et al. (2004) and Feifer and Ornstein (2004).
- Alignment of protocols supporting the roll-out of the best practice at all levels (organisational alignment), is confirmed by Waterman et al. (2007).

- Besides policies and protocols, other structures such as budget and infrastructure that supports the implementation and roll-out of the best practice should be aligned at all levels.

To ensure the successful adoption of best practices at every institution, it is crucial that there is a general advance plan for implementation and roll-out of best practices (Nolan, Schall, Erb, \& Nolan, 2005). This plan should preferably be driven by national policy and aligned with and supported at all levels. In this regard, Berg et al. (2005) found a discrepancy between what was intended to happen according to the research cycle of implementation and what actually happened at the individual hospitals in the provinces. An advance plan will prevent the haphazard implementation and roll-out of best practices, and ensure equal accessibility and quality of health care, which is a fundamental patient right, outlined in the National Patients' Rights Charter (National Department of Health, 2007). Furthermore, to enhance ownership, leaders at all levels should be involved in the developing, pilot-testing and implementing the roll-out plan.

Leadership for change at all levels as requirement was found to be crucial to successfully roll-out best practices. However, participants noted a lack of leadership. The importance of the motivating, convincing and advisory role of a leader to facilitate the roll-out of best practices was emphasised (Edwards \& Grinspun, 2011; Pearson, Upenieks, Yee, \& Needleman, 2008). However, it remains unclear what leader characteristic(s) and role(s) of the leader influences the rollout of a best practice most, and which types of the leader and the organisation fostering leadership should prioritise to enhance the roll-out of best practices (National Health Service Institute for Innovation and Improvement, 2010).

The literature confirms a variety of supporting structures, essential for rolling-out best practices. These are (1) resources (Edwards \& Grinspun, 2011; Pearson et al., 2008), (2) good communication regarding the best practice and its implementation (Pearson et al., 2008), (3) organisational structure (Edwards \& Grinspun, 2011), and (4) education and development (Edwards \& Grinspun, 2011). Finally, overall findings suggest that support and coordination should preferably come from authority levels, for example officials at national and provincial levels, who must in turn provide support to the management of organisations and individual staff. Management can support at an individual level. For example, officials at national level can provide the policy and support for the roll-out of best practices, and officials at provincial level can ensure that the policy is known, provide the budget, drive the change and appoint leaders (Bergh et al., 2007).

The requirements for roll-out were found to be interrelated. For example, leadership should be engaged in planning the dissemination (National Health Service Institute for Innovation and Improvement, 2010), and supporting and reinforcing structures should be aligned with best practices. The requirements for roll-out should thus be viewed as a collaborative effort. Notwithstanding that participants mentioned one requirement (such as supporting and 
reinforcing structures) was mentioned more by participants, than another requirement (such as roll-out plan) it is unclear how the existence, or lack of one or more of the requirements influences the roll-out of best practices.

\subsection{Implications of the study}

Understanding the requirements to successfully roll-out KMC in South Africa has important implications for the future rollout of best practices in South Africa. The contextual nature of the requirements to successfully roll-out best practices, were based on the KMC experience, which makes this study unique in the field of implementation science. Plan for the roll-out of best practices must integrate planning for alignment, leadership for change and reinforcing and supporting structures. The study's findings can be used in the roll-out process of best practices by clinicians, and policy makers and decision makers in the South African health care system. Organisations planning to roll-out a best practice could use the findings as a guide to develop an operational plan to facilitate smoother roll-out of best practices.

\section{Conclusions, limitations \& recommendations for future research}

Conclusions, limitations and recommendations of the study are discussed next.

\subsection{Conclusions}

The roll-out of best practices is a complex process and requires collaboration by key stakeholders at all levels of the health care system. The participants in our study identified and described four requirements for rolling-out KMC which were congruent with the four benefit levers identified by Edwards and Grinspun (2011) (alignment, plan for roll-out, leadership for change, and supporting and reinforcing structures). Although the requirements for roll-out were found at different levels of the health care system, the participants did not mention certain requirements at each specific level. In general, the requirements identified by Edwards and Grinspun should therefore be used in planning for the roll-out of best practices.

\subsection{Limitations}

A limitation of this study was that participants came from only 12 institutions in seven provinces. It was not possible to recruit more participants, since some did not respond to the invitation. In addition, some provinces were more intensively involved with implementing and rolling-out KMC than others. As a result, the researcher was challenged to obtain sufficient participants from all provinces.

\subsection{Recommendations for future research}

Finally, a more in-depth, qualitative exploration of each requirement, including what leadership characteristics and roles and support influence the roll-out of best practices. The influence of the existence, or lack of one or more of the requirements for roll-out should also be investigated by means of comparison studies and controlled trials. Research is also needed to promote fuller understanding of how to devise and apply the requirements in the wider adoption of best practices in the South African health care setting. Furthermore, health system research is required to create a better understanding of the influence of a system on the roll-out of best practices, and what is needed in the system to enhance the roll-out of best practices in the South African health context.

\section{Authors' contributions}

WTH conducted the study, collected data, carried out the analyses, wrote the paper. CSM and SJCvdW supervised the study, reviewed the manuscript and approved the final version.

\section{Acknowledgements}

We are grateful to all the health professionals who participated in this study, to $\mathrm{xxx}$ who co-coded the data, and to the National Research Foundation and The university is NorthWest University who provided financial support in the form of bursaries.

\section{REFERENCES}

Bergh, A. M., Arsalo, I., Malan, A. F., Patrick, M., Pattinson, R. C., \& Phillips, N. (2005). Measuring implementation progress in kangaroo mother care. Acta Paediatrica, 94(8), 1102-1108.

Bergh, A. M., Davy, K., Van Rooyen, E., Manu, R., Greenfield, J., \& Participants from Ghana Health Service. (2009). Scaling up kangaroo mother care in Ghana. In Proceedings of the 28th Conference on Priorities in Perinatal Care in South Africa. Drakensberg: Perinatal Priorities.

Bergh, A. M., \& Pattinson, R. C. (2003). Development of a conceptual tool for the implementation of kangaroo mother care. Acta Pcediatrica, 92(6), 709-714.

Bergh, A. M., Rogers-Bloch, Q., Pratomo, H., Uhudiyah, U., Poernomo, I., Sidi, S. S., et al. (2012). Progress in the implementation of kangaroo mother care in 10 hospitals in Indonesia. Journal of Tropical Pediatrics, 58(5), 402-405.

Bergh, A. M., Van Rooyen, E., Lawn, J., Zimba, E., Ligowe, R., \& Ciundu, G. (2007). Retrospective evaluation of kangaroo mother care practices in Malawian hospitals. Pretoria: Ministry of Health.

Bergh, A.-M., Van Rooyen, E., \& Pattinson, R. C. (2008). Scaling up kangaroo mother care in South Africa: 'on-site' versus 'offsite' educational facilitation. Human Resources for Health, 6(13), $1-6$.

Bodenheimer, T. (2007). The science of spread: How innovations in care become the norm. California: California Health Care Foundation.

Botma, Y., Greeff, M., Mulaudzi, F. M., \& Wright, S. C. D. (2010). Research in health sciences (1st ed). Cape Town: Pearson Education, 370 p.

Chin, M. H., Cook, S., Drum, M. L., Jin, L., Guillen, M., Humikowski, C. A., et al. (2004). Improving diabetes care in Midwest community health centers with the health disparities collaborative. Diabetes Care, 27(1), 2-8. 
Edwards, N., \& Grinspun, D. (2011). Understanding whole systems change in healthcare: The case of emerging evidence-informed nursing service delivery models. Ottowa: Canadian Health Services Research Foundation.

Feifer, C., \& Ornstein, S. M. (2004). Strategies for increasing adherence to clinical guidelines and improving patient outcomes in small primary care practices. Joint Commission Journal on Quality and Patient Safety, 30(8), 432-441.

Grol, R. (2001). Successes and failures in the implementation of evidence-based guidelines for clinical practice. Medical Care, 39(8), II-46-II-54.

Grol, R., \& Grimshaw, J. (2003). From best evidence to best practice: effective implementation of change in patients' care. Lancet, 362(9391), 1225-1230.

Harrison, M. B., Legare, F., Graham, I. D., \& Fervers, B. (2010). Adapting clinical practice guidelines to local context and accessing barriers to their use. Canadian Medical Association Journal, 182(2), E78-E84.

Institute for Healthcare Improvement. (2008). 5 million lives campaign-getting started kit: Rapid response teams. Cambridge: Institute for Healthcare Improvement.

King, N., \& Horrocks, C. (2010). Interviews in qualitative research (1st ed, pp. 144-163). London, United Kingdom: SAGE Publications.

Lawn, J. E., Mwansa-Kambafwile, J., Horta, B. L., Barros, F. C., \& Cousens, S. (2010). Kangaroo mother care' to prevent neonatal deaths due to preterm birth complications. International Journal of Epidemiology, 39(Supplement 1), i144-i154.

National Department of Health. (2007). The patients' rights charter. Pretoria: National Department of Health.

National Department of Health. (2012). Strategic plan for Maternal, Newborn, Child and Women's Health (MNCWH) and nutrition in South Africa 2012-2016. Pretoria: National Department of Health.
National Health Service Institute for Innovation and Improvement. (2010). Improving healthcare quality at scale and pace lessons from the productive ward: Releasing time to care ${ }^{\mathrm{TM}}$ programme full report. London: National Health Service.

Nolan, K., Schall, M. W., Erb, F., \& Nolan, T. (2005). Using a framework for spread: the case of patient access in the veterans health administration. Joint Commission Journal on Quality and Patient Safety, 31(6), 339-347.

Nyqvist, K. H., Anderson, G. C., Bergman, N., Cattaneo, A., Charpak, N., Davanzo, R., et al. (2010). Towards universal kangaroo mother care: recommendations and report from the First European conference and Seventh International Workshop on kangaroo mother care. Acta Paediatrica, 99(6), $820-826$.

Oxford English Dictionary. (2015). Precise. Oxford: Oxford University Press. London: Oxford.

Pattinson, R. C., Arsalo, I., Bergh, A.-M., Malan, A. F., Patrick, M., \& Phillips, N. (2005). Implementation of kangaroo mother care: a randomized trial of two outreach strategies. Acta Pcediatrica, 94(7), 924-927.

Pearson, M. L., Upenieks, V., Yee, T., \& Needleman, J. (2008). Spreading nursing unit innovation in large hospital systems. The Journal of Nursing Administration, 38(3), 146-152.

Porter, M. E., \& Teisberg, E. O. (2007). How physicians can change the future of health care. The Journal of the American Medical Association, 297(10), 1103-1111.

Simons, H. (2009). Case study: research in practice (1st ed). London: SAGE Publications, $190 \mathrm{p}$.

Waterman, H., Marshall, M., Noble, J., Davies, H., Walshe, K., Sheaff, R., et al. (2007). The role of action research in the investigation and diffusion of innovations in health care, the PRIDE project. Quality Health Research, 17(3), 373-381. 